British Journal of Nutrition (2022), 127, 1599

doi: $10.1017 / \mathrm{S} 0007114521005249$

(C) The Author(s), 2022. Published by Cambridge University Press on behalf of The Nutrition Society. This is an Open Access article, distributed under the terms of the Creative Commons Attribution licence (https://creativecommons.org/licenses/by/4.0/), which permits unrestricted re-use, distribution, and reproduction in any medium, provided the original work is properly cited.

\title{
Corrigendum
}

\section{Patient-Generated Subjective Global Assessment (PG-SGA) predicts length of hospital stay in lung adenocarcinoma patients - CORRIGENDUM}

Jilu Lang, Yanan Shao, Jiehao Liao, Jia Chen, Xuewen Zhou, Rong Deng, Wei-Jan Wang and Xian Sun

(First published online 10 September 2021)

DOI: https://doi.org/10.1017/S0007114521003500

This article was published with incorrect corresponding author details.

Currently reads: *Corresponding author: Xian Sun, email sunshine1d1@126.com

This should read: *Corresponding authors: Xian Sun, email sunshine1d1@126.com and Wei-Jan Wang, email: cvcsky@gmail.com 